\title{
Bioengineered human acellular vessels for dialysis access in patients with end-stage renal disease: two phase 2 single-arm trials
}

\begin{abstract}
Jeffrey H Lawson, Marc H Glickman, Marek Ilzecki, Tomasz Jakimowicz, Andrzej Jaroszynski, Eric K Peden, Alison J Pilgrim, Heather L Prichard, Malgorzata Guziewicz, Stanisław Przywara, Jacek Szmidt, Jakub Turek, Wojciech Witkiewicz, Norbert Zapotoczny, Tomasz Zubilewicz, and Laura E Niklason

Humacyte, Durham, NC, USA (J H Lawson MD PhD, H L Prichard PhD, A J Pilgrim MD PhD, M H Glickman MD); Duke University, Durham, North Carolina, USA (J H Lawson); Sentara Heart Hospital, Norfolk, VA, USA (M H Glickman); Department of Vascular Surgery and Angiology (M Ilzecki MD PhD, S Przywara MD PhD, T Zubilewicz MD PhD), Department of Family Medicine (A Jaroszynski MD PhD), Medical University of Lublin, Lublin, Poland; Department of General, Vascular and Transplant Surgery, Medical University of Warsaw, Warsaw, Poland (T Jakimowicz MD PhD, J Szmidt MD PhD); Research and Development Centre, Vascular Surgery Department, General Hospital in Wrocław, Wrocław, Poland (M Guziewicz MD PhD, J Turek MD, W Witkiewicz MD PhD, N Zapotoczny MD); Cardiovascular Surgery Associates, Houston, TX, USA (E K Peden MD); and Departments of Anesthesia \& Biomedical Engineering, Yale University, New Haven, CT, USA (L E Niklason MD PhD)
\end{abstract}

\section{Summary}

Background-For patients with end-stage renal disease who are not candidates for fistula, dialysis access grafts are the best option for chronic haemodialysis. However, polytetrafluoroethylene arteriovenous grafts are prone to thrombosis, infection, and intimal hyperplasia at the venous anastomosis. We developed and tested a bioengineered human acellular vessel as a potential solution to these limitations in dialysis access.

Methods-We did two single-arm phase 2 trials at six centres in the USA and Poland. We enrolled adults with end-stage renal disease. A novel bioengineered human acellular vessel was implanted into the arms of patients for haemodialysis access. Primary endpoints were safety (freedom from immune response or infection, aneurysm, or mechanical failure, and incidence of adverse events), and efficacy as assessed by primary, primary assisted, and secondary patencies at 6 months. All patients were followed up for at least 1 year, or had a censoring event. These trials are registered with ClinicalTrials.gov, NCT01744418 and NCT01840956.

Correspondence to: Dr Laura E Niklason, Departments of Anesthesia \& Biomedical Engineering, Yale University, Room 301D Amistad Building, New Haven, CT 06519, USA, laura.niklason@yale.edu.

Contributors

LEN, JHL, and AJP wrote the report, and HLP provided data analysis. JHL, MHG, MI, TJ, AJ, MG, EKP, SP, JS, JT, WW, NZ, and TZ implanted grafts and followed up patients.

Declaration of interests

LEN, AJP, HLP, and JHL own stock or stock options in Humacyte. AJP and MHG work as consultants for Humacyte. The other authors declare no competing interests. 
Findings-Human acellular vessels were implanted into 60 patients. Mean follow-up was 16 months (SD 7.6). One vessel became infected during 82 patient-years of follow-up. The vessels had no dilatation and rarely had post-cannulation bleeding. At 6 months, $63 \%$ (95\% CI 47-72) of patients had primary patency, 73\% (57-81) had primary assisted patency, and 97\% (85-98) had secondary patency, with most loss of primary patency because of thrombosis. At 12 months, 28\% (17-40) had primary patency, 38\% (26-51) had primary assisted patency, and 89\% (74-93) had secondary patency.

Interpretation-Bioengineered human acellular vessels seem to provide safe and functional haemodialysis access, and warrant further study in randomised controlled trials.

Funding-Humacyte and US National Institutes of Health.

\section{Introduction}

Expanded polytetrafluoroethylene (ePTFE) grafts are often used in patients receiving haemodialysis who are not candidates for autologous fistula creation or whose fistula has failed. However, infection, intimal hyperplasia, and thrombosis of implanted grafts often lead to failure and abandonment, and contribute to the high cost of renal replacement therapy. ${ }^{1-4}$ Indeed, $90 \%$ of patients with graft thrombosis have intimal hyperplasia that contributes to stenosis, ${ }^{3}$ and infection is reported in up to $17 \%$ of synthetic arteriovenous grafts. ${ }^{5}$ Many biological alternatives to ePTFE for dialysis access have been studied, but none has gained wide acceptance. Bovine carotid artery grafts 6,7 have been shown to provide acceptable patency in only small, single-centre studies, and depopulated bovine ureters and bovine mesenteric vein are prone to stenosis and aneurysm. ${ }^{8-13}$ Cryopreserved human vein shows some tendency for dilatation and could increase panel-reactive antibodies, ${ }^{14}$ whereas a previous autologous tissue-engineered conduit was subject to structural degradation and aneurysm formation. ${ }^{15,16}$ Although these various xenogeneic, allogeneic, and autologous grafts have been studied for haemodialysis access, none has performed convincingly better than ePTFE.

We cultured human acellular vessels in vitro using human vascular smooth muscle cells that are cultured on a biodegradable polymer. ${ }^{17-19}$ Decellularisation of the vessels removes all living cells while retaining extracellular matrix proteins and preserving the mechanical properties of the graft. ${ }^{18,20}$ In baboons, human acellular vessels implanted as arteriovenous grafts have functioned as intended, without mechanical degradation and with patency of more than $80 \%$ for up to 6 months. ${ }^{18}$ We did two phase 2 trials to study the safety and efficacy of these vessels in 60 patients requiring haemodialysis. Enrolment was completed in June 2014, and each patient has been followed up for at least 1 year, or had a censoring event.

\section{Methods}

\section{Study design and participants}

We did this single-group trial at three hospitals in Poland and three in the USA. We enrolled patients older than 18 years with end-stage renal disease and deemed not suitable for fistula creation in the opinion of the operating surgeon. We excluded patients with more than one 
previous ePTFE graft in the operative limb, severe peripheral vascular disease in either arm, known or suspected central vein obstruction on the implantation side, or those who required a graft that would cross the elbow. We also excluded patients with severe cardiac disease (New York Heart Association Functional Class III or IV), myocardial infarction or stroke within the preceding 6 months, ventricular tachyarrhythmias or unstable angina, active cancer in the previous year, or life expectancy of less than 1 year. In the USA, we also excluded candidates on a waiting list for renal transplantation. The goal of these exclusion criteria was to ensure that most patients would survive to the end of follow-up, since patients who need dialysis have an average life expectancy of only 5 years from the initiation of haemodialysis. Because most patients had had several failed accesses by the time of enrolment, the corresponding life expectancy of these patients would be substantially shorter than 5 years.

The US and Polish studies had different maximum age allowed ( 75 years in Poland, 80 years in the USA), and different time to first dialysis cannulation permitted for the human acellular vessels ( 8 weeks for all patients except the final ten patients in Poland, where it was 4 weeks). In addition, Polish patients received low molecular weight heparin as postoperative prophylaxis for 4 weeks.

Both studies were done in accordance with good clinical practice (ISO14155 and ECH E6/E8/E2A) and the Declaration of Helsinki. The US study was done as an Investigative New Drug (number 15263). The ethics committee or institutional review board of each participating clinical centre approved the protocol, and each patient provided written informed consent before enrolment.

\section{Procedures}

Human acellular vessels, $6 \mathrm{~mm}$ in diameter and $35-42 \mathrm{~cm}$ in length, were produced in vitro by methods similar to those previously described (figure 1). ${ }^{17,19,21}$ Briefly, human vascular smooth muscle cells were derived from deceased organ and tissue donors, aged 18-50 years, meeting eligibility requirements for all relevant communicable diseases, including seronegativity for HIV and hepatitis and no history of intravenous drug use. ${ }^{22}$ Smooth muscle cells were isolated by explant methods, with 1 million-60 million cells isolated from each donor. After expansion, we seeded the cells at 1 million per $\mathrm{cm}$ onto polyglycolic acid polymer scaffolds (Biomedical Structures; Warwick, RI, USA) that were contained within flexible, single-use bioreactors (Charter Medical; Winston-Salem, NC, USA; figure 1). Developing vessels were subjected to pulsatile cyclic distension for 8 weeks, and were then decellularised. We decellularised the vessel to remove immunogenic cellular antigens while preserving the comparatively non-immunogenic extracellular matrix constituents, including collagens type I and III, fibronectin, and vitronectin. ${ }^{18}$

The human acellular vessels used in this study were off-white tubular structures (figure 1) with a mean wall thickness of $440 \mu \mathrm{m}$ (SD 85; in 97 vessels), mean suture retention strength of $210 \mathrm{~g}$ (SD $53 \mathrm{~g}$; in 109 vessels), and mean burst strength of $2914 \mathrm{~mm} \mathrm{Hg}$ (SD 928; in five vessels). Histological evaluation by haematoxylin and eosin staining showed a proteinaceous structure without visible nuclei, and with voids in the extracellular matrix where smooth muscle cells had resided before decellularisation. Masson's trichrome staining showed that 
most of the extracellular matrix in the vessels was collagen, with a mean hydroxyproline content of $76 \mathrm{mg} / \mathrm{g}$ dry tissue (SD 9; in 97 vessels). ELISA showed mean residual beta-actin protein of $59 \mathrm{ng} / \mathrm{cm}$ human acellular vessel length (SD 25; in 98 vessels), and less than 300 $\mathrm{pg} / \mathrm{cm}$ human acellular vessel length of MHC I (in 52 vessels). Gel electrophoresis showed no DNA bands greater than 200 base pairs at a sensitivity of $1.25 \mathrm{ng}$. The mechanically robust vessels were thus primarily comprised of collagens and other associated extracellular matrix proteins, with very low levels of residual cellular materials. We subjected each batch of human acellular vessels to these mechanical and compositional assays before release for clinical use.

We implanted the first vessel on Dec 5, 2012, and the last on June 3, 2014. In all cases, the vessels were implanted in the upper arm (above the elbow) by standard vascular surgical techniques. In 59 patients, the grafts were placed as a brachial-to-axillary straight graft, and in one patient the graft was placed in an axillary-to-axillary loop configuration. If available, intraoperative ultrasound was used to confirm vessel patency. Otherwise, patency was confirmed after surgery by clinical and hand-held Doppler examination. Aspirin 75-325 mg per day, given orally, was started after surgery (in the USA) or on cessation of subcutaneous low molecular weight heparin (in Poland), and continued for the duration of follow-up.

Follow-up visits were done at 4-7 days (USA only), 15 days, monthly until 6 months, and then at 9 months, 12 months, 18 months, and 24 months. At all visits, we assessed the vessel clinically. From 15 days onwards, we did Doppler ultrasound imaging of the entire implant. Interventions to maintain or restore graft patency, and other adverse events, were recorded at each visit. Laboratory assessments (haematological, clinical chemistry, and coagulation studies) were done at 2 weeks, 4 weeks, 8 weeks, 12 weeks, and 26 weeks (in Poland) or at 4-7 days, 2 weeks, 4 weeks, 8 weeks, 12 weeks, and 26 weeks (in the USA). Panel-reactive antibodies were assessed at 2 weeks, 4 weeks, 8 weeks, 12 weeks, and 26 weeks (Poland) or at 4 weeks, 12 weeks, 26 weeks, and 104 weeks (USA). We used a customised ELISA to test for the presence of $\mathrm{IgG}$ antibodies that bind to the solubilised vessel material before implantation, on the same timetable as assessments of panel-reactive antibodies. The IgG ELISA used a monoclonal antibody against human $\mathrm{IgG}$ in a sandwich-type reaction with a colorimetric readout, and we used pooled human sera as a negative control. Dialysis cannulation was permitted using the human acellular vessel after the 8-week visit (the first 30 Polish patients and all US patients) or after the 4-week visit (final ten Polish patients). We selected initiation of cannulation at 4-8 weeks on the basis of preclinical findings regarding cellular repopulation of human acellular vessels. Patients continued to use the human acellular vessel for dialysis three times per week throughout follow-up, until vessel abandonment. Primary safety and efficacy endpoints were at 6 months, and we have followed up all patients for at least 12 months, unless the patients were censored or the vessel was abandoned before 12 months.

\section{Outcomes}

The primary endpoints were safety (absence of immune response or infection, aneurysm, or mechanical failure, and incidence of adverse events), and efficacy as assessed by primary, primary assisted, and secondary patencies at 6 months. Secondary endpoints were patency 
and interventions at 12 months, 18 months, and 24 months, and changes in panel-reactive antibodies following implantation.

\section{Statistical analysis}

We defined primary patency as functional access patency until any type of intervention to maintain or restore patency; primary assisted patency was defined as a vessel still functioning without occurrence of thrombosis; and secondary patency was defined as functional access patency, with or without preceding successful interventional or surgical procedures to maintain or reestablish patency, until either final failure or until the vessel was abandoned. ${ }^{23}$ Key parameters were summarised descriptively. There were minor protocol deviations relating to entry laboratory test values that were just outside the permitted range, and to visits made outside the protocol-specified windows, usually because the patient was admitted to hospital for unrelated medical problems. We describe the efficacy of the vessels in terms of time to loss of patency using the Kaplan-Meier method ${ }^{23}$ with separate assessments for primary, primary assisted, and secondary patency. Patients were censored at death, renal transplantation, or study withdrawal.

\section{Role of the funding source}

Humacyte designed the studies, and (working with physicians at the clinical sites) collected, analysed, and interpreted the data, and wrote the report. The decision to submit for publication was the responsibility of employees of Humacyte, as well as consultants to Humacyte. JHL, HLP, AJP, and LEN had access to all of the data.

\section{Results}

We implanted vessels in 40 patients in Poland and 20 in the USA. Baseline characteristics of the two study populations were similar (table 1), although the proportion of female patients and the incidence of diabetes, and cardiovascular, cerebrovascular, and peripheral arterial disease were higher in the USA than in Poland. In both studies, patients had multiple previous accesses for haemodialysis, ranging from one to nine in Poland, and one to six in the USA (table 1). Mean follow-up was 16 months (SD 7.6), with a cumulative follow-up of 82 patient-years. During follow-up, eight patients were censored (including four who died while the vessel was patent, one transplantation, and three who withdrew consent). The one patient who underwent renal transplantation after vessel implantation had a well-functioning renal allograft 9 months after transplantation. One of the 60 vessels was abandoned after 2 weeks because of ischaemic monomelic neuropathy in that arm, and the remainder were patent at the scheduled assessment at 4-8 weeks. In Poland, 37 of 40 patients started dialysis via the human acellular vessel within 2 weeks of the scheduled timepoint, and in the USA 17 of 19 vessels were deemed suitable for dialysis at 8 weeks.

At 6 months, the pooled primary patency was 63\% (95\% CI 47-72), primary assisted patency was 73\% (57-81), and secondary patency was 97\% (85-98; table 2, figure 2). At 12 months, primary patency was $28 \%$ (17-40), primary assisted patency was $38 \%$ (26-51), and secondary patency was $89 \%$ (74-93). Mean blood flow through the implanted vessels measured by ultrasound was typically more than $1.0 \mathrm{~L}$ per min (table 2 ) and was stable over 
time. Mean luminal diameters of the vessels remained stable, without evidence of substantial dilatation or narrowing (table 2). At 18 months, primary patency was 18\% (9-30), primary assisted patency was 30\% (17-43), and secondary patency was 81\% (66-90). At 24 months, among the nine patients who were followed up for at least that long, primary patency was $15 \%$ (6-27), primary assisted patency was $25 \%$ (12-40), and secondary patency was $80 \%$ (61-87).

Interventions to maintain or restore patency were done at a rate of 1.89 per patient-year (table 2). Most interventions were thrombectomies, which were associated with loss of primary and primary assisted patency. In Poland, a thrombosed graft is typically treated first with a simple thrombectomy, and a stent or other intervention used only if repeated thromboses occur. By contrast, in the USA, imaging and stenting or angioplasty along with thrombectomy are common, thereby decreasing the chance of re-thrombosis. This difference in clinical practice might underlie the higher intervention rate in Poland than in the USA (table 2). The number of venous anastomotic reconstructions ( $\mathrm{n}=16$; a rate of 0.20 per patient-year) is consistent with previous reports. ${ }^{24}$ One patient had acute steal syndome and underwent banding of the acellular vessel within 1 day of implantation.

Ten human acellular vessels were abandoned (ie, had lost secondary patency), ranging from 2 weeks to 85 weeks after implantation. Of these, five were abandoned in the first year after implantation. Two abandonments occurred in patients in whom the vessel had been overdilated with 8-mm high-pressure balloons in attempts to treat stenosis, which might have disrupted the 6-mm diameter acellular vessel material. One human acellular vessel was ligated and resected to manage suspected ischaemic monomelic neuropathy, while the others were abandoned mostly because of recurrent stenosis of the outflow vein or venous anastomosis, with associated thromboses. Examinations for an association of patient age, sex, or cause of end-stage renal disease with graft abandonment showed no clear trends (data not shown). No human acellular vessel was abandoned because of infection of the vessel itself.

Four patients died during follow-up. None of the deaths were attributable to the human acellular vessel, and the mortality rate was consistent with that reported for the general population of patients who need haemodialysis. ${ }^{25}$

There were no instances of acute perioperative vessel infection and no cases of delayed infection with purulent contamination (as can occur with ePTFE implants) requiring total graft excision. There were three vascular access site infections, ${ }^{26}$ one of which was an infected haematoma that resolved with intravenous antibiotics, and one of which was an infected ePTFE segment that had been used to reconstruct the venous anastomosis, but which did not infect the vessel material. The third infection involved the human acellular vessel itself, in the cannulation zone, where a perigraft haematoma became cellulitic with positive blood cultures, and was treated by resection and reconstruction of the affected vessel segment (followed by successful dialysis using the human acellular vessel).

155 serious adverse events occurred in 33 patients, most of which were interventions to maintain or restore vessel patency. Aside from these interventions, the episode of steal 
syndrome, and infections, there were no serious adverse events attributable to the human acellular vessel. Haematological tests, clinical chemistry, and coagulation profiles did not change substantially after vessel implantation (data not shown), and bleeding events after cannulation were rare.

There was no evidence of systemic immune or inflammatory response to the human acellular vessels. Assay for serum IgG antibodies against the vessel material showed increases from baseline in only seven patients, and in two of these patients the titre doubled and then returned to baseline. In the remaining five patients, the $\operatorname{IgG}$ increases resolved within a few months and did not correlate with graft inflammation or dilatation, nor were they correlated with interventions to maintain graft patency. In the five patients with increased $\mathrm{IgG}$, one patient retained primary patency, one had primary assisted patency, three had secondary patency, and no patient had their vessel abandoned.

Panel reactive antibodies, for both MHC-I and MHC-II, increased in the 6 months after implantation in three patients (data not shown). In two of these patients, failure of a renal allograft or withdrawal of systemic immunosuppression for a previous allograft were temporally related to the rise. This association implicated the development of panel reactive alloantibodies to a rejecting renal allograft, rather than to the acellular vessel. In a third patient, MHC-I panel reactive antibodies increased 34\% from baseline 3 months after implantation, without apparent cause and without clinical signs of vessel rejection.

We recorded no true aneurysms of the human acellular vessels. The diameters of the vessels remained generally stable over follow-up as measured by ultrasound imaging (table 2). There were two occasions in which ultrasound suggested a vessel diameter of around 12 $\mathrm{mm}$, but in both cases the diameter had returned to normal at the subsequent assessment, suggesting spurious observations or resolution of small pseudoaneurysms. 14 pseudoaneurysms occurred in ten patients, and were generally associated with needle cannulation sites. Pseudoaneurysms were typically detected by routine ultrasound monitoring and in most cases were not clinically significant. Two pseudoaneurysms required surgical intervention.

We procured a small segment of vessel material for histological assessment from eight patients at 16-55 weeks after implantation. Seven segments were from the venous anastomotic region and one was from the central part of the vessel, and all were taken during otherwise-indicated surgical revision procedures.

The vessel explant obtained at 16 weeks after implantation near the venous anastomosis had evidence of repopulation by monocytic CD68 cells (figure 3A). The localisation of CD68 cells correlates approximately with the location of smooth muscle actin positive cells, indicating that some repopulating cells had a smooth muscle or myofibroblast phenotype (figure 3B). The abluminal concentration of CD68 and smooth muscle actin cells implies that repopulation of the vessel wall occurred primarily from the surrounding connective tissues. Staining for CD31, a marker of vascular endothelium, was positive on the vessel lumen and was seen in the vessel suggesting re-endothelialisation (figure 3C). Staining for CD20 (B-lymphocytic marker) and CD3 (T-lymphocytic marker) was absent in most 
specimens, and presented as scattered individual cells in two specimens, suggesting little adaptive immune response to the implanted vessels.

The latest vessel explant, taken at 55 weeks and also near the venous anastomosis, had fewer CD68 cells than the sample taken at 16 weeks (figure 3D). By contrast, smooth muscle actin was expressed throughout the vessel wall at 55 weeks (figure 3E), and CD31 staining appeared on the lumen (figure 3F). These results support an ongoing and progressive remodelling of the vessel with tissue-specific vascular cells. Unlike native veins, remodelled implanted vessels did not contain appreciable elastin, and had thicker walls than native vein of similar diameters.

A mid-graft segment that was explanted at 44 weeks included several cannulation tracts (figure $3 \mathrm{G}, \mathrm{H}$ ), including a recent cannulation site with fresh clot extending into the tract from the lumen (haematoxylin and eosin stain; figure 3G). Another section showed a partly healed cannulation tract (figure $3 \mathrm{H}$ ), and many of these cells also expressed smooth muscle actin (not shown). This mid-graft segment also had evidence of CD31 staining on the implanted vessel lumen, indicating endothelialisation (figure 3I). Thus, implant remodelling seems to have occurred from both the luminal and abluminal surfaces of the implanted vessel, and patient cells appear to re-infiltrate cannulation tracts.

\section{Discussion}

Regenerative medicine has progressed significantly in the past 15 years, with many tissues and prototype organs in development. ${ }^{27-29}$ Whereas previous engineered vascular grafts for haemodialysis access have required autologous biopsies from the patient to obtain cells to grow the graft, ${ }^{15}$ the vessels used in these studies were grown from banked human vascular cells, then decellularised to produce an acellular vessel that can be implanted into any patient, without a patient biopsy or tissue matching.

The practice of fistula and graft placement for haemodialysis access varies in different geographical regions and in different countries. Generally, patients older than 70 years and who have many comorbidities often fail to mature their fistulas, as do women who are of small stature. In those patients, it is not uncommon to place an ePTFE graft rather than attempt to make a fistula. Patients in our study came from several backgrounds: those who had failed fistula and who needed a graft; those who were never suitable for fistula and who needed a graft; and those who had failed both fistula and grafts, and who needed another graft.

The implanted vessels, the first engineered, decellularised vascular prostheses, had no clinical or ultrasound evidence of structural degeneration, or true aneurysm formation, over a mean follow-up of 16 months in 60 patients. Although we recorded 14 pseudoaneurysms, cannulation-associated pseudoaneurysms are a well known complication of repeated needle access in both dialysis fistulas and grafts. The incidence of pseudoaneurysm ranges from 5\% to more than $60 \%,{ }^{30-32}$ making 14 pseudoaneurysms in ten patients within the reported range. 
Secondary patency of $97 \%$ at 6 months and $89 \%$ at 1 year was higher than that reported in multicentre studies of ePTFE (55-65\% at 1 year). ${ }^{33-35}$ The prevalence of comorbid diseases was similar in the Polish cohort and the United States Renal Data System for patients with end-stage renal disease, including for hypertension ( $85 \%$ vs 86\%), diabetes (38\% vs 44\%), and peripheral vascular disease (13\% vs $13 \%$; table 1). ${ }^{25}$ By contrast, these comorbidities were more common in the US cohort (hypertension 100\%, diabetes 55\%, peripheral vascular disease 35\%), implying that the US cohort had a higher disease burden than average patients with end-stage kidney disease in the USA. Thus, the cohorts in these studies were not enriched for patients with few comorbidities.

Previous autologously tissue engineered arteriovenous grafts resulted in two of nine grafts having dilatation that necessitated partial or complete graft removal. ${ }^{15}$ Other biological grafts such as bovine mesenteric vein and depopulated ureters also have substantial rates of dilatation. ${ }^{8,11}$ By contrast, we recorded no true aneurysms in the implanted vessels at any time. The small perigraft haematomas or pseudoaneurysms we report were probably cannulation site injuries caused by needle access, and are consistent with all forms of both prosthetic and autogenous vascular access. They were identified through protocol-required ultrasound assessments and were generally of little clinical significance because the access remained patent and supported haemodialysis.

The high secondary patency of the implanted vessels was primarily due to the rarity of terminal vessel loss caused by infection or rethrombosis. Patients were maintained on aspirin to decrease the risk of platelet activation by the exposed collagen substrate. The intervention rate of 1.89 interventions per patient-year is consistent with rates for ePTFE grafts, ${ }^{35,36}$ meaning that secondary patency of the vessels was not maintained via heroic interventional measures. Primary patency was similar to that reported for ePTFE, ${ }^{3,33}$ and most loss of primary patency was because of graft thrombosis. Although primary patency is a key clinical endpoint in evaluation of arteriovenous grafts, the high rates of secondary patency confer both clinical and economic benefits by reducing the need for new access placement, which is more complex and expensive than rescuing an access already in situ.

The host response to the implanted vessel material might underlie some of the increase in secondary patency compared with ePTFE. Pre-clinical studies ${ }^{37}$ of decellularised engineered grafts in pigs showed that infiltrating host cells had less activation of the mTOR pathway than did autologous vein grafts 4 weeks after implantation. Because the mTOR pathway is central to intimal hyperplastic responses and occlusion after arterial grafting or stenting, decreased activation of this pathway by human acellular vessels could contribute to improved long-term patency.

No evidence of immune rejection of the vessel was detected from longitudinal analysis of serum samples, nor in histological explants. In two of the three patients with increased panel-reactive antibodies, the increase coincided with cessation of immunosuppressive drugs from a previous rejected renal transplantation, which can cause such increases. ${ }^{38}$ The generally minimal effect on panel-reactive antibodies compares favourably with cryopreserved human vein, for which the average increase is around $80 \% .{ }^{14}$ The quantitative decellularisation of the vessels, which reduces cellular antigens including MHC-I to very 
low levels, is probably responsible for the lack of an adaptive immune response to the implant.

Positive immunostaining for markers of smooth muscle cells and endothelial cells in repopulated grafts means that tissue-appropriate cells become invested into the matrix, converting the vessel into a living tissue. Population of the vessel wall with cells that express markers of both monocytes (CD68) and smooth muscle cells (smooth muscle actin) is consistent with studies of vascular remodelling. Mesenchymal precursors expressing monocytic markers such as CD68 have been shown to be involved in pulmonary vascular remodelling and atherosclerotic disease. ${ }^{39,40}$ Thus, there could be a phenotypic continuum of cells that repopulate the vessel.

There was only one infection that required resection of a segment of the vessel in 82 patientyears. This infection rate (1.3\% per patient-year) is lower than that reported with ePTFE, and is similar to arteriovenous fistulas $(0.9 \%))^{5,41}$ Because the vessel extracellular matrix repopulates with host cells, it may be more resistant to bacterial infection than ePTFE, which inhibits efficient chemotaxis of host neutrophils. ${ }^{42}$

These results, representing the most comprehensive assessment of any bioengineered vascular tissue to date, should be interpreted within specific limitations. This is an interim report of two phase 2 studies done at a small number of selected centres, and there was no randomised comparator graft or fistula. Furthermore, the different effect, if any, of key comorbidities between the two study populations was not directly evaluated or controlled for in the analyses. Because candidates for kidney transplantation were excluded only from the US study, the US patients might have had a greater disease burden than those in Poland.

In conclusion, the human acellular vessels seem to be safe, well tolerated, and have no significant immunogenic potential. The vessel seems to be durable, withstanding repeated cannulation over periods greater than 1 year without aneurysm or structural degradation. After implantation, the vessel repopulates and remodels with host cells and might have a resistance to infection only reported previously for native arteriovenous fistulas.

Furthermore, in this limited number of patients (60), the implant functioned as a suitable conduit for dialysis access and did not require a prolonged time for maturation. These observations will require testing in a larger, prospective, randomised, controlled, clinical study.

\section{Acknowledgments}

This work was supported by Humacyte and by the US National Institutes of Health (R44 HL118736). We thank Michael Perelman and Shannon Dahl for assistance with manuscript preparation. William Tente and Juliana Blum assisted with site recruitment and regulatory documents. Justin Strader and Stuart Irwin oversaw graft manufacture, Robert Schutte developed the manufacturing system, and Maribel Santiago did histological analysis. Sally Wade provided editorial assistance.

\section{References}

1. Roy-Chaudhury P, Kelly BS, Miller MA, et al. Venous neointimal hyperplasia in polytetrafluoroethylene dialysis grafts. Kidney Int. 2001; 59:2325-34. [PubMed: 11380837] 
2. Schon D, Blume SW, Neibauer K, Hollenbeak CS, de Lissovoy G. Increasing the use of arteriovenous fistula in hemodialysis: economic benefits and economic barriers. Clin J Am Soc Nephrol. 2007; 2:268-76. [PubMed: 17699424]

3. Dixon BS, Beck GJ, Vazquez MA, et al. Effect of dipyridamole plus aspirin on hemodialysis graft patency. N Engl J Med. 2009; 360:2191-201. [PubMed: 19458364]

4. Haskal ZJ, Trerotola S, Dolmatch B, et al. Stent graft versus balloon angioplasty for failing dialysisaccess grafts. N Engl J Med. 2010; 362:494-503. [PubMed: 20147715]

5. Akoh JA, Patel N. Infection of hemodialysis arteriovenous grafts. J Vasc Access. 2010; 11:155-58. [PubMed: 20175060]

6. Harlander-Locke M, Jimenez JC, Lawrence PF, et al. Bovine carotid artery (artegraft) as a hemodialysis access conduit in patients who are poor candidates for native arteriovenous fistulae. Vasc Endovascular Surg. 2014; 48:497-502. [PubMed: 25487247]

7. Kennealey PT, Elias N, Hertl M, et al. A prospective, randomized comparison of bovine carotid artery and expanded polytetrafluoroethylene for permanent hemodialysis vascular access. J Vasc Surg. 2011; 53:1640-48. [PubMed: 21609797]

8. Das N, Bratby MJ, Shrivastava V, et al. Results of a seven-year, single-centre experience of the longterm outcomes of bovine ureter grafts used as novel conduits for haemodialysis fistulas. Cardiovasc Intervent Radiol. 2011; 34:958-63. [PubMed: 21360240]

9. Smith MP, Spark JI, Patel JV, Border DF, Oswal D, Lewington AJ. Massive aneurysmal dilatation of a depopulated ureteric hemodialysis xenograft. Hemodial Int. 2009; 13:3-5. [PubMed: 19210270]

10. Kingston GT, Darby CR, Roberts IS. The pathology of depopulated bovine ureter xenografts utilized for vascular access in haemodialysis patients. Histopathology. 2009; 55:154-60. [PubMed: 19694822]

11. Hatzibaloglou A, Velissaris I, Kaitzis D, Grekas D, Avdelidou A, Kiskinis D. ProCol vascular bioprosthesis for vascular access: midterm results. J Vasc Access. 2004; 5:16-18. [PubMed: 16596534]

12. Manduz S, Katrancioglu N, Ozker E, Dogan K. Early thrombosis of bovine mesenteric vein grafts after infrainguinal reconstruction. Int J Angiol. 2008; 17:37-39. [PubMed: 22477370]

13. Schmidli J, Savolainen H, Heller G, et al. Bovine mesenteric vein graft (ProCol) in critical limb ischaemia with tissue loss and infection. Eur J Vasc Endovasc Surg. 2004; 27:251-53. [PubMed: 14760592]

14. Madden R, Lipkowitz G, Benedetto B, Kubanov A, Miller M, Bow L. Decellularized cadaver vein allografts used for hemodialysis access do not cause allosensitization or preclude kidney transplantation. Am J Kidney Dis. 2002; 40:1240-43. [PubMed: 12460043]

15. McAllister TN, Maruszewski M, Garrido SA, et al. Effectiveness of haemodialysis access with an autologous tissue-engineered vascular graft: a multicentre cohort study. Lancet. 2009; 373:144046. [PubMed: 19394535]

16. Wystrychowski W, McAllister TN, Zagalski K, Dusserre N, Cierpka L, L’Heureux N. First human use of an allogeneic tissue-engineered vascular graft for hemodialysis access. J Vasc Surg. 2014; 60:1353-57. [PubMed: 24103406]

17. Niklason LE, Gao J, Abbott WM, et al. Functional arteries grown in vitro. Science. 1999; 284:48993. [PubMed: 10205057]

18. Dahl SLM, Kypson AP, Lawson JH, et al. Readily available tissue-engineered vascular grafts. Sci Transl Med. 2011; 3:ra9.

19. Poh M, Boyer M, Solan A, et al. Blood vessels engineered from human cells. Lancet. 2005; 365:2122-24. [PubMed: 15964449]

20. Dahl SLM, Koh J, Prabhakar V, Niklason LE. Decellularized native and engineered arterial scafolds for transplantation. Cell Transplant. 2003; 12:659-66. [PubMed: 14579934]

21. Niklason LE, Abbott WA, Gao J, et al. Morphologic and mechanical characteristics of bovine engineered arteries. J Vasc Surg. 2001; 33:628-38. [PubMed: 11241137]

22. US Food and Drug Administration. CFR - Code of Federal Regulations Title 21, Part 1271: Human Cells, Tissues and Cellular and Tissue-Based Products. http://www.accessdata.fda.gov/ scripts/cdrh/cfdocs/cfcfr/CFRSearch.cfm?CFRPart=1271 (accessed April 24, 2016) 
23. Sidawy AN, Gray R, Besarab A, et al. Recommended standards of reports dealing with arteriovenous hemodialysis accesses. J Vasc Surg. 2002; 35:603-10. [PubMed: 11877717]

24. Miller PE, Carlton D, Deierhoi MH, Redden DT, Allon M. Natural history of arteriovenous grafts in hemodialysis patients. Am J Kidney Dis. 2000; 36:68-74. [PubMed: 10873874]

25. United States Renal Data System. Annual Data Report. 2015. http://www.usrds.org/adr.aspx (accessed March 20, 2016)

26. CDC. Dialysis Event Protocol. http://www.cdc.gov/nhsn/PDFs/pscManual/ 8pscDialysisEventcurrent.pdf (accessed March 20, 2016)

27. Shinoka T, Shum-Tim D, Ma PX, et al. Creation of viable pulmonary artery autografts through tissue engineering. J Thorac Cardiovasc Surg. 1998; 115:536-46. [PubMed: 9535439]

28. Lancaster MA, Knoblich JA. Organogenesis in a dish: modeling development and disease using organoid technologies. Science. 2014; 345:1247125. [PubMed: 25035496]

29. Raya-Rivera AM, Esquillano D, Fierro-Pastrana R, et al. Tissue-engineered autologous vaginal organs in patients: a pilot cohort study. Lancet. 2014; 384:329-36. [PubMed: 24726478]

30. Valenti D, Mistry H, Stephenson M. A novel classification system for autogenous arteriovenous fistula aneurysms in renal access patients. Vasc Endovascular Surg. 2014; 48:491-96. [PubMed: 25487245]

31. Fokou M, Teyang A, Ashuntantang G, et al. Complications of arteriovenous fistula for hemodialysis: an 8-year study. Ann Vasc Surg. 2012; 26:680-84. [PubMed: 22534263]

32. Zibari GB, Rohr MS, Landreneau MD, et al. Complications from permanent hemodialysis vascular access. Surgery. 1988; 104:681-86. [PubMed: 3175866]

33. Huber TS, Carter JW, Carter RL, Seeger JM. Patency of autogenous and polytetrafluoroethylene upper extremity arteriovenous hemodialysis accesses: a systematic review. J Vasc Surg. 2003; 38:1005-11. [PubMed: 14603208]

34. Katzman HE, Glickman MH, Schild AF, Fujitani RM, Lawson JH. Multicenter evaluation of the bovine mesenteric vein bioprostheses for hemodialysis access in patients with an earlier failed prosthetic graft. J Am Coll Surg. 2005; 201:223-30. [PubMed: 16038820]

35. Lok CE, Sontrop JM, Tomlinson G. Cumulative patency of contemporary fistulas versus grafts (2000-2010). Clin J Am Soc Nephrol. 2013; 8:810-18. [PubMed: 23371955]

36. Nassar GM, Glickman MH, McLafferty RB, et al. A comparison between the HeRO graft and conventional arteriovenous grafts in hemodialysis patients. Semin Dial. 2014; 27:310-18. [PubMed: 24428351]

37. Quint C, Kondo Y, Manson RJ, Lawson JH, Dardik A, Niklason LE. Decellularized tissueengineered blood vessel as an arterial conduit. Proc Natl Acad Sci USA. 2011; 108:9214-19. [PubMed: 21571635]

38. Augustine JJ, Woodside KJ, Padiyar A, Sanchez EQ, Hricik DE, Schulak JA. Independent of nephrectomy, weaning immunosuppression leads to late sensitization after kidney transplant failure. Transplantation. 2012; 94:738-43. [PubMed: 22955228]

39. Frid MG, Brunetti JA, Burke DL, et al. Hypoxia-induced pulmonary vascular remodeling requires recruitment of circulating mesenchymal precursors of a monocyte/macrophage lineage. Am J Pathol. 2006; 168:659-69. [PubMed: 16436679]

40. Allahverdian S, Chehroudi AC, McManus BM, Abraham T, Francis GA. Contribution of intimal smooth muscle cells to cholesterol accumulation and macrophage-like cells in human atherosclerosis. Circulation. 2014; 129:1551-59. [PubMed: 24481950]

41. Schild AF, Perez E, Gillaspie E, Seaver C, Livingstone J, Thibonnier A. Arteriovenous fistulae vs. arteriovenous grafts: a retrospective review of 1,700 consecutive vascular access cases. J Vasc Access. 2008; 9:231-35. [PubMed: 19085891]

42. Chang CC, Lieberman SM, Moghe PV. Quantitative analysis of the regulation of leukocyte chemosensory migration by a vascular prosthetic biomaterial. J Mater Sci Mater Med. 2000; 11:337-44. [PubMed: 15348014] 


\section{Research in context}

\section{Evidence before this study}

We searched MEDLINE and Cochrane databases between Jan 1, 2000, and Oct 1, 2015, for the terms: human engineered dialysis vessel, xenogeneic arterial graft, and allogeneic vascular dialysis graft. We identified 242 reports in English in MEDLINE and 137 in Cochrane, of which ten were included after review for relevance. For patients with endstage renal disease who are not candidates for autogenous fistula creation, synthetic grafts are the best option for haemodialysis access. Expanded polytetrafluoroethylene (ePTFE) grafts are routinely used, but infection, intimal hyperplasia, and thrombosis often lead to failure and abandonment, resulting in temporary catheter use and overall higher costs. Human-derived alternatives might dilate and be immunogenic (cryopreserved human vein) or suffer structural degradation and aneurysm formation (autologous, tissue engineered conduit). None of the various xenogeneic, allogeneic, or engineered autologous grafts previously assessed for haemodialysis have performed decisively better than ePTFE.

\section{Added value of this study}

These studies are the first to evaluate the safety and efficacy of human acellular vessels in patients requiring haemodialysis, and are the largest to be done for any tissue engineered vascular conduit. In the two studies, $63 \%$ of patients had primary patency, $73 \%$ had primary assisted patency, and $97 \%$ had secondary patency at 6 months; and 27\%,36\%, and $89 \%$, respectively, at 12 months. Interventions to maintain or restore patency were similarly common to those for ePTFE, and infections were rare. The implanted vessels did not show dilatation, had adequate blood flow for haemodialysis, and typically did not have post-cannulation bleeding after dialysis. After implantation, the vessels repopulated with host vascular cells.

\section{Implications of all available evidence}

Bioengineered human acellular vessels could provide safe and functional haemodialysis access, and warrant further study in randomised controlled trials. 


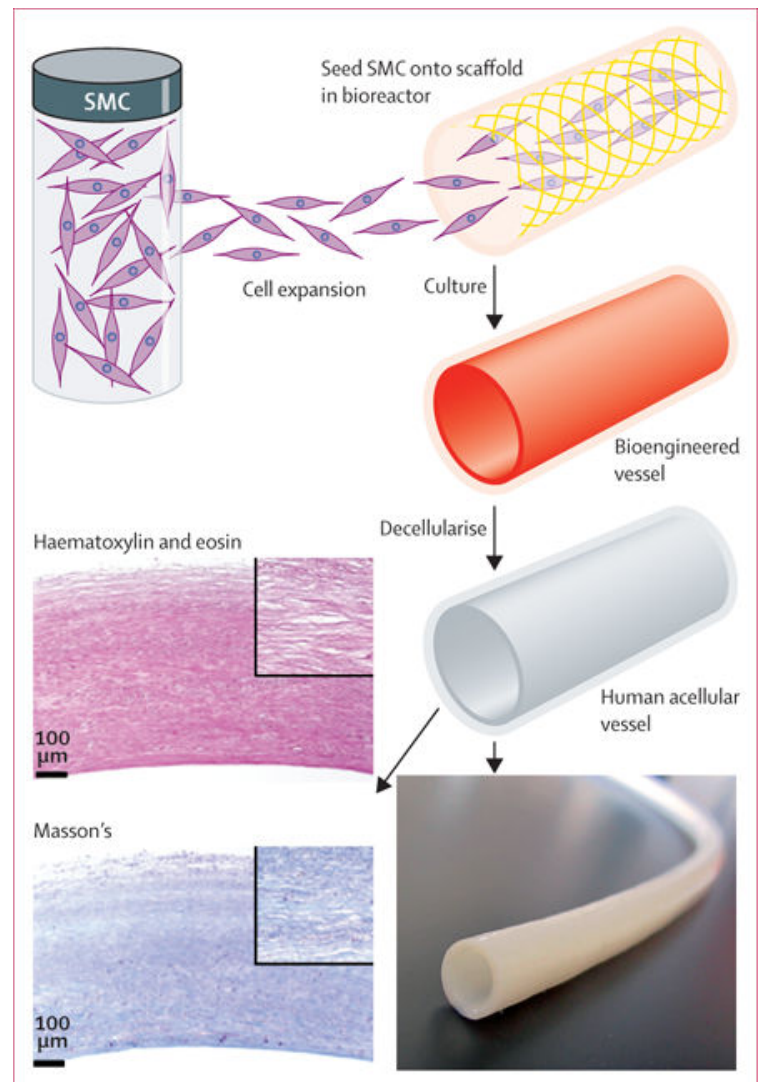

Figure 1. Production of arteriovenous grafts

Smooth muscle cells are seeded onto a biocompatible scaffold within a single-use bioreactor. During culture, a cellular bioengineered vessel is grown, which is then decellularised to produce the human acellular vessel. Gross appearance is an off-white uniform tubular structure, and haematoxylin and eosin stain and Masson's trichrome stain show dense extracellular matrix without cellular or nuclear remnants. SMC=smooth muscle cell. 


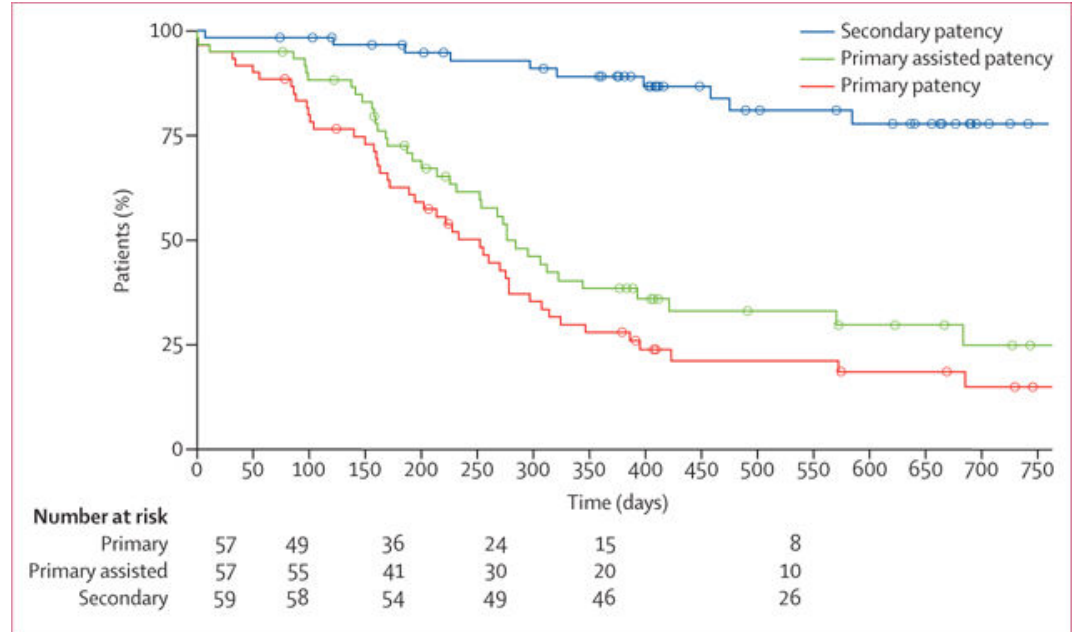

Figure 2. Kaplan-Meier curves for human acellular vessel patencies

Data from the USA and Poland were combined for this analysis. 

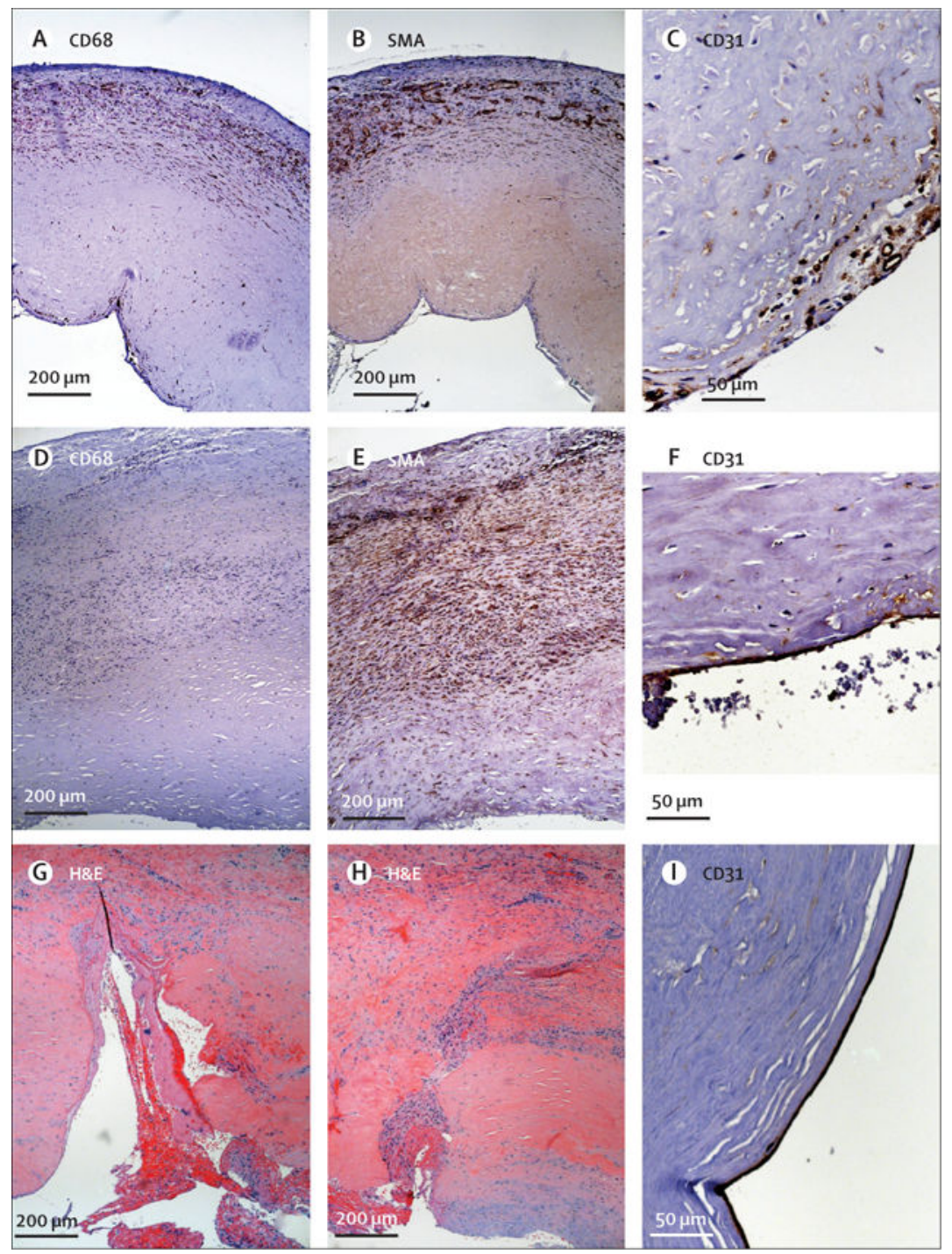

$\underline{50 \mu \mathrm{m}}$

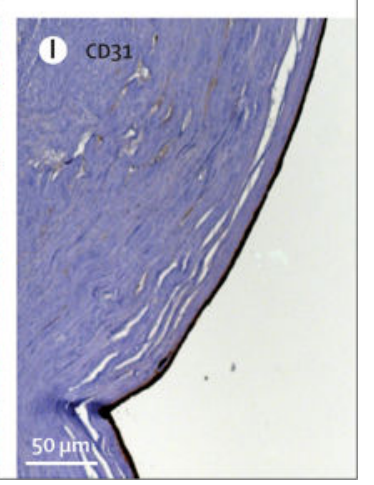

Figure 3. Human acellular vessel remodelling

Immunoperoxidase histology staining of explant at 16 weeks shows abluminal repopulation by (A) CD68 positive cells; (B) smooth muscle actin positive cells; and (C) CD31 positive cells on the graft lumen. Haematoxylin counterstain shows nuclei. Immunoperoxidase histological staining of explant at 55 weeks shows (D) few CD68 positive brown cells; (E) extensive smooth muscle actin positive cell repopulation extending almost throughout the graft wall; and (F) CD31 positive staining on the graft lumen. Histological assessment of midgraft segments resected at 44 weeks because of infected perigraft haematoma shows two previous cannulation sites. Haematoxylin and eosin staining shows $(G)$ a very recent cannulation site with fresh clot in the cannulation tract; $(\mathrm{H})$ a healing cannulation site with cell repopulation occurring from the luminal surface. (I) CD31 immunoperoxidase stain shows luminal positive cells in this mid-vessel segment. For all panels, the vessel lumen is at the bottom of the panel. SMA=smooth muscle actin. $\mathrm{H} \& \mathrm{E}=$ haematoxylin and eosin. 


\section{Table 1}

\section{Baseline characteristics}

\begin{tabular}{llc} 
& $\begin{array}{l}\text { Polish cohort } \\
(\mathbf{n = 4 0 )}\end{array}$ & $\begin{array}{l}\text { US cohort } \\
(\mathbf{n = 2 0})\end{array}$ \\
\hline Mean age (years; SD) & $59(10)$ & $61(8)$ \\
\hline $\begin{array}{l}\text { Mean duration of follow-up (months; SD) } \\
\quad 16 \text { months }\end{array}$ & $18 \cdot 5(6 \cdot 4)$ & $10 \cdot 0(7 \cdot 0)$ \\
$\quad 212$ months & $39(98 \%)$ & $14(70 \%)$ \\
$\geq 18$ months & $36(90 \%)$ & $11(55 \%)$ \\
$\quad 24$ months & $23(58 \%)$ & $4(20 \%)$ \\
\hline Men & $9(23 \%)$ & 0 \\
\hline Race & $22(55 \%)$ & $7(35 \%)$ \\
White & $40(100 \%)$ & $6(30 \%)$ \\
African American & 0 & $13(65 \%)$ \\
Other & 0 & $1(5 \%)$ \\
\hline Comorbidities & & \\
Hypertension & $34(85 \%)$ & $20(100 \%)$ \\
Diabetes & $15(38 \%)$ & $11(55 \%)$ \\
Cardiovascular disease & $13(33 \%)$ & $13(65 \%)$ \\
Cerebrovascular disease & $2(5 \%)$ & $8(40 \%)$ \\
Peripheral arterial disease & $5(13 \%)$ & $7(35 \%)$ \\
Venous thromboembolic disease & $3(8 \%)$ & $2(10 \%)$ \\
\hline Aspirin use & $3 \cdot 6(2 \cdot 1)$ & $3 \cdot 6(2 \cdot 2)$ \\
\hline Mean number of previous dialysis accesses (SD) & & \\
\hline & & $17(85 \%)$ \\
\hline
\end{tabular}

Data are $\mathrm{n}(\%)$ unless stated otherwise. 
Table 2

\section{Clinical results}

\begin{tabular}{|c|c|c|}
\hline & Polish cohort $(n=40)$ & US cohort $(n=20)$ \\
\hline Adverse events & 232 & 178 \\
\hline Serious adverse events & 91 & 64 \\
\hline Deaths & 1 & 3 \\
\hline Withdrawals & 1 & 2 \\
\hline Transplantations & 1 & 0 \\
\hline Delayed haemostasis after dialysis & 3 & 8 \\
\hline Pseudoaneurysms & 10 (in seven patients) & 4 (in three patients) \\
\hline Aneurysm & 0 & 0 \\
\hline Steal syndrome requiring intervention & 1 & 1 \\
\hline Human acellular vessel abandonment & 6 & 4 \\
\hline \multicolumn{3}{|l|}{ Patency $(\%, 95 \%$ CI $)$} \\
\hline \multicolumn{3}{|l|}{ Primary } \\
\hline 6 months & $70 \%(53-82)$ & $46 \%(23-67)$ \\
\hline 12 months & $33 \%(20-49)$ & $13 \%(2-34)$ \\
\hline \multicolumn{3}{|l|}{ Primary assisted patency } \\
\hline 6 months & $78 \%(61-88)$ & $61 \%(35-79)$ \\
\hline 12 months & $41 \%(26-56)$ & $31 \%(10-55)$ \\
\hline \multicolumn{3}{|l|}{ Secondary patency } \\
\hline 6 months & $100 \%(100-100)$ & $89 \%(64-97)$ \\
\hline 12 months & $95 \%(81-99)$ & $76 \%(48-90)$ \\
\hline \multicolumn{3}{|l|}{ Interventions } \\
\hline Total procedures & 125 & 30 \\
\hline Thrombectomy & 98 & 18 \\
\hline Angioplasty & 36 & 19 \\
\hline Revision & 14 & 2 \\
\hline Removal or ligation & 0 & 2 \\
\hline Interventions per patient-year & 1.95 & $1 \cdot 67$ \\
\hline \multicolumn{3}{|l|}{ Infections } \\
\hline Haematoma or ePTFE infection & 1 & 1 \\
\hline Human acellular vessel infection & 0 & 1 \\
\hline \multicolumn{3}{|l|}{ Mean flow rate (mL per min; SD) } \\
\hline Day 1 & $1544(890)$ & $705(493)$ \\
\hline Week 12 & $1825(1161)$ & $1439(861)$ \\
\hline
\end{tabular}




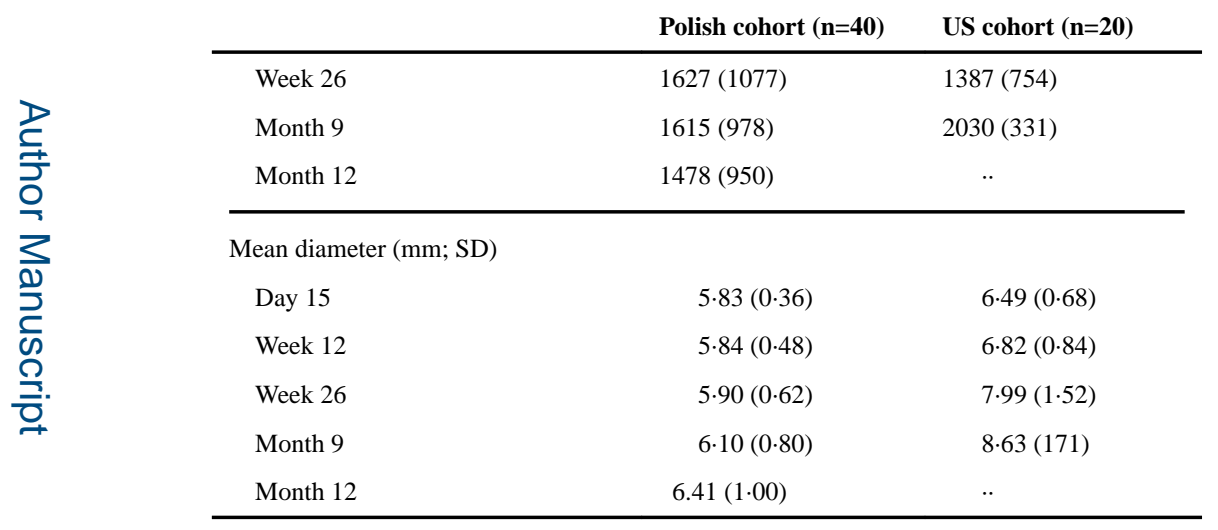

Data are n, or n (\%) unless stated otherwise. ePTFE=expanded polytetrafluoroethylene. 